\title{
The position of random autokinetic movement and the physiological position of rest are frequently stable and identical
}

\author{
VAEGAN \\ School of Psychology, University of New South Wales, Kensington, N.S.W., 20\$9, Australia
}

\begin{abstract}
Both the autokinetic illusion (AKI) and involuntary eye movements when a fixation point goes off have been attributed to unmonitored drift eye movements which result from constant features of the oculomotor system. Pilot studies confirmed that the visual directions where these effects had no directional bias, the position of random autokinetic movement (PRAKM) and the physiological position of rest (PPR), were highly correlated and reliable. The more precise main experiment showed that they were usually, but not always, stable and identical. Drifts are therefore not totally due to stable features of the oculomotor system but are a compound of several slow responses which can differ with both stimulus conditions and time. They are not all equally unmonitored.
\end{abstract}

Many experiments, reviewed in Levy (1972), show that the direction the eye moves involuntarily when an AKI stimulus is turned off is opposite the previously reported AKI direction. This suggests that the undetected slow components of drift presumed to cause both effects are identical. Another large body of evidence show's that AKI direction reports in a given situation are highly reliable, suggesting that these undetected slow components are due to permanent forces in the oculomotor system. In this paper, the previous findings are confirmed but the stronger hypotheses do not hold universally.

\section{The Physiological Position of Rest (PPR)}

The eye muscles contain over $50 \%$ by weight fibrous elastic tissue. With no innvervation, as in deep sleep, anaesthesia, or death, the eye turns to a stable equilibrium position called the anatomical position of rest (Adler, 1959). In the dark, an alert person cannot maintain fixation direction. Innervation adds to the purely mechanical forces to produce the physiological position of rest (PPR) (Duke-Elder, 1949).

Skavenski and Steinman (1970) show that initial drifts of $\mathbf{3 0} \mathrm{min}$ of arc occur consistently in the same direction when the fixation point is turned off. Eye position then varies over about $1 / 3^{\circ}$ around this new mean for some time when constant refixation saccades are allowed. When saccades are suppressed, the eye drifts steadily until it is out of recording range. They do not know if it reaches any equilibrium. Nachimas (1961) doubted that it did. We can now show that it does.

If a fixation point is turned on again after a short

This research was conducted while the author was a postgraduate student at Monash University, Melbourne, Australia. The author has changed his name from John Levy to Vaegan and no longer uses any name but the latter. delay, during which they try to hold their eyes still in various positions, subjects report that the second point generally appears to be more peripheral than the first. About $60 \%$ of the variance of such location errors can be accounted for by eye movements (Matin. Pearce, Matin \& Kibler, 1966). ${ }^{1}$ Matin and Kibler (1966) suggests that by varying stimulus positions horizontally or vertically, and asking subjects to make a two-alternative forced-choice judgment of the second stimulus position relative to the first (left/ right or up/down, respectively), a stimulus position can be found at which each response is equiprobable.

Although they never attempted to do so themselves, Levy (1969) has successfully developed the method. He called the visual direction, operationally defined in this way, the physiological position of rest (PPR) because in this position the eye of a living person is most relaxed. Rest does not imply quiescence. Normal eye movements would simply be reduced and without any directional basis. Duke-Elder's (1949) concept of the PPR was similar, but he knew of no measure. The position is different in each eye, often far from straight ahead, and typically convergent and downturned (Ebenholtz \& Wolfson, 1975; Levy, 1969).

\section{The Position of Random Autokinetic Movement (PRAKM)}

A recent general review of the measures, theories, and data of autokinetic illusion research (Levy, 1972) urges that direction reports are involved in all meaningful measures. It recommends the PRAKM measure outlined here.

Autokinesis direction reports to a stimulus straight ahead are consistent for any individual (Pearce, 1966), but at other positions stimuli are reported to move in the direction of eye turn with increasing 
probability as a function of angle (Carr, 1910). PRAKM, like PPR, can therefore be defined independently in each primary meridian as that stimulus position where both responses are equally probable when subjects make two-alternative forced-choice reports of autokinesis direction.

To reduce variance and increase reliability. eye of viewing. posture, and aftereffects from previous eye positions must be carefully controlled (Levy, 1968, 1973). With staircase psychophysics, stimuli are kept close to PRAKM. Short interstimulus intervals and long viewing times before any response also reduce aftereffects and increase the likelihood that only the current stimulus position determines the response. In these circumstances. PRAKM had been measured with a mean staircase range of $22^{\circ}$ and an estimated standard error of less than $5^{\circ}$ (Levy, 1968, 1973). ${ }^{2}$

\section{PREVIOUS STUDIES}

Some of the earliest and most extensive studies of autokinesis (Adams, 1912; Carr. 1910) report that for most eyes there was a stimulus position at which reported AKI direction was random. It was so stable it was used consistently through a long series of experiments as the zero eye-turn position for that eye of that subject. In 1907. Carr showed that there was a similar position at which a fixated point source appeared to move in all directions equiprobably during blinking. Ginsborg and Maurice (1959) also measured a position of random "blink-induced" movement. They, too. say it does not change. This position may therefore be a third way of measuring an equilibrium position for the eye. None of these studies used systematic psychophysics or experimental designs.

In a previously unpublished study, while developing the methods reported here, Levy (1968) showed that even crude PRAKM and PPR measures were reliable in eight subjects. The test-restest rank-order correlations (tau) (Siegal, 1956) for both measures in both dimensions shown in Table 1 are all significant $(p<.05)$. In a further eight subjects, PRAKM and PPR were significantly correlated in both the horizontal and vertical (tau was 0.572 and 0.642 , respectively). Figure 1 is a two-dimensional scatter plot in which the coincidence of these two measures can be seen directly. The relationship is only rough, because the measures used are now known to be

Table 1

Rank Order Corr elation Coefficients for Eight Subjects

Using Less Pre cise Measures Than Those Reported Here (Levy, 1968)

\begin{tabular}{lcc} 
& Horizontal & Vertical \\
\hline PRAKM & .715 & .946 \\
PPR & .545 & .715 \\
\hline
\end{tabular}

Note - In all cases $p(t a u=0)<.05$.



Figure 1. Scatter plot of PRAKM and PPR positions, each measured in both horizontal and vertical for eight sabjects. From Levy (1968).

biased by aftereffects and other procedurally induced errors.

\section{METHOD}

\section{Design}

If autokinesis direction and eye movements after a light goes off are both related directly to slow components of drift which are stable features of the oculomotor system of each particular eye, we can predict that PRAKM and PPR should be not merely reliable and correlated but stable and identical. A correlation design cannot test these much stronger predictions. Estimates of degree of common variance over a set of subjects are insufficient. Independent decisions need to be made every time parameters are compared within each subject.

As outlined above, both measures (PRAKM and PPR) are based on a two-alternative forced-choice task in which stimulus positions are varied until each response is equiprobable. The prediction that they are stable and identical is therefore equivalent to saying that for either task. once a position where both responses are equiprobable has been found, responses to both that task and the other will always be equiprobable at that position. ${ }^{3}$

The sequential probability ratio rest (SPRT) (Wetherill, 1966) was chosen because it is the most efficient method of testing a hypothesis that responses occur with a stated probability $\left(\mathbf{H}_{\mathbf{0}}\right)$ against other hypotheses that the probabilities are some other distinct values $\left(\mathrm{H}_{1}\right.$ and $\left.\mathrm{H}_{1}\right)$. That is, the SPRT minimizes the average sample number for a test of any given accuracy and power. Depending on whether the test is one- or two-tailed, the difference between the probabilities (P) and the probability of Type 1 and Type 2 errors ( $a$ and $\beta$, respectively) that one is prepared to tolerate, boundaries can be specified on a graph of the cumulative frequency of excess responses against total responses. Using only one stimulus value, responses are collected continuously till a decision area is reached, as shown in Figure 2. Each SPRT is therefore essentially an independent experiment.

Using the SPRT forces experimenters to formulate and justify Type 2 error rates and alternative hypotheses which are implicit and incidental but vital consequences of an arbitrarily chosen sample number in many fixed sample size experiments. Type 1 error rate $\left(\mathrm{P}_{a}=0.01\right)$ was set strictly because it adds confidence to 


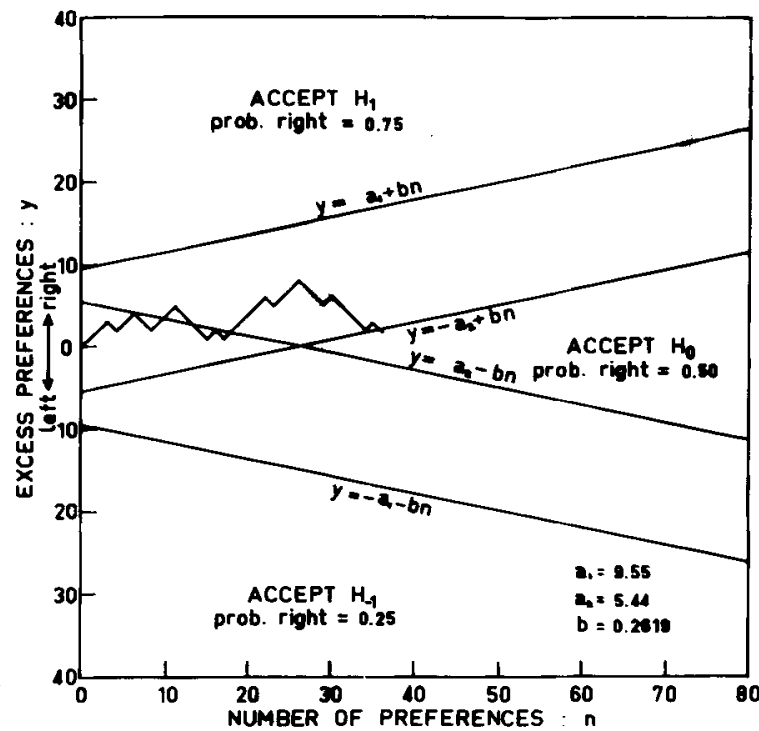

Figure 2. A typical SPRT, showing the boundary conditions and their equations as specified in Armitage (1960).

any difference that is found and it has small sampling cost above the 0.05 rate. Type 2 error rate was more traditional $\left(P_{\beta}=0.05\right)$ because there was less concern about it. The boundary, or size of difference in response probability $(P)$, between $H_{O}$ and alternative hypotheses $H_{1}$ and $H_{1}$ which will be considered significant is more difficult to specify. It should be as small as possible. Consequently, it was decided to take as large an average sample as subjects could tolerate and let that determine $P$. The main limitation, then, is that the expected sample size distribution is positively skewed and occasionally very long runs occur, tasking subjects' endurance.

For a two-tailed test with $a=0.01, \beta=0.05, \mathrm{H}_{0}=0.5$, and $P$ $=0.25$, tables by Armitage $(1960)$ showed that the average sample number when $\mathrm{H}_{\mathrm{O}}$ was in fact true would be 33 , i.e., $14 \mathrm{~min}$ testing. This meant minimum average first-session testing times of $11 / 2 \mathrm{~h}$. Since these were considered very tight alternative hypothesis boundaries for subjects to remain within, given the current degree of experimental control possible, and as this minimum time is as long as was practicable, these boundary conditions were considered more than sufficiently stringent.

\section{Apparatus}

All aspects of the test situation apart from stimulus timing and instructions which modify the judgments required of subjects are identical for the two measures. To control postural effects and interocular differences, the subject sat in a barber's chair and bit onto a molar biteboard fixed to the chair. A headrest, locked behind the skull, further restricted head movement. An occluder. mounted near the biteboard, blocked the left eye. The right eye was thus held almost at the center of rotation of the chair, which could be rotated, raised, or lowered. At the subject's right hand was a box containing a self-centering telephone key which could be positioned so that the subject could move it left/right or up/down. The response registered on the experimenter's control panel in the next room.

\section{Subjects}

The 12 subjects were volunteers and off-campus acquaintances of the experimenter. They had not previously experienced the autokinetic illusion and were never told that the stimuli they were judging did not truly shift till after the experiment, when the hypothesis under test was also explained. None wore glasses for distance viewing. Three, who could not return for the second session, had to be replaced.

\section{Procedure}

When the subject first arrived, the perimeter was hidden by a curtain. A biteboard was made, the subject was fitted into a chair. and the height of the perimeter was adjusted so that it was level with his right eye. There were four possible starting measures (PRAKM or PPR, H or V). Three subjects were assigned to each condition. In each subject studied, only one parameter (PRAKM or PPR) in one dimension (horizontal or vertical) was used as a baseline measure because of the long testing times involved. All tests of identity and stability were made relative to that baseline.

The nature of the stimulus. decision, and response were explained before the subject was finally locked into the biteboard, the light extinguished, the curtain drawn aside, and the experimenter started the stimulus sequence from the next room. Whenever the experimenter entered or left the room, the curtain was drawn before the lights were turned on.

The point where either response was equiprobable was estimated by Wetherill's $(1 \% 66)$ routine seventeen, a double random staircase technique. Staircases started from $0^{\circ}$ and ran with $5^{\circ}$ steps till six reversals. The mean of the six reversals was calculated and a second pair of staircases immediately began, starting from the stimulus position nearest this mean, and finished after eight reversals. ${ }^{2}$ Then the experimenter returned to the subject's room and moved either the chair or the perimeter until one stimulus was precisely at the mean of these eight reversals. He told the subject that, despite the adjustment, the task would not change. A third pair of staircases then ran from the selected position and continued till 10 reversals. Again, the subject or the perimeter was adjusted so that the mean of these 10 reversals was immediately ahead of his eye. He was told that nothing else would change.

Actually, at this stage, the first SPRT was run, to test whether the stimulus position defined by this third mean would still cause both responses to occur equiprobably when the stimulus no longer moved in staircase fashion. It was called the first stability test. Only 2 of these 12 first stability tests failed to accept $\mathrm{H}_{0}$, and the errors were small in these two cases. The position was reestimated by a further 10 reversal double random staircase and a second SPRT run to ensure that the two were in agreement before proceeding.

Throughout the experiment, whenever an SPRT failed to accept $\mathrm{H}_{\mathrm{o}}$, a new measure was taken to estimate the size of the position difference. Only after stability tests was a second SPRT made, because only here was it essential for the two measures to agree before an identity test could meaningfully be made.

When the stability test and final staircase mean were in agreement, the alternative task, stimulus pattern, and decision required were described to the subject. Those whose PRAKM had been measured were given a PPR task, and vice versa. The dimension of the judgment (left/right or up/down) was unchanged. Another SPRT called the first identity test was run with this task. The stimulus was in the position where the stability test and final staircase mean agreed. If it failed to accept $\mathbf{H}_{\mathbf{0}}$, a staircase was run to estimate the extent of the difference, but there was no further SPRT.

At least a week later, the subjects returned and the procedure was repeated from the beginning of the stability test. The stimulus at which the previous staircase and identity test had agreed was again the starting point. These two tests were called the second stability and identity tests.

\section{RESULTS}

Previous experience had shown that the mean staircase range was the same for PRAKM and PPR and was a good index of variability. The mean range found here $\left(23.2^{\circ}\right)$ was almost as good as any previously achieved and suggests a standard error of measurement of less than $5^{\circ}$. This is also consistent with the variability between the three preliminary 
Table 2

Original Measurement and Estimated Errors in Those Cases Where $H_{\circ}$ Was Rejected, Expressed as Degrees from Straight Ahead (Right and Up Turns Are Positjve)

\begin{tabular}{|c|c|c|c|c|c|c|c|}
\hline \multicolumn{4}{|c|}{ Preliminary Staircase } & \multicolumn{4}{|c|}{ SPRT } \\
\hline Subject & Task & Dimension & Mean & $\begin{array}{c}\text { First } \\
\text { Stability }\end{array}$ & $\begin{array}{c}\text { First } \\
\text { Identity }\end{array}$ & $\begin{array}{r}\text { Second } \\
\text { Stability }\end{array}$ & $\begin{array}{l}\text { Second } \\
\text { Identity }\end{array}$ \\
\hline 1 & PRAKM & $\mathbf{H}$ & .0 & -6.5 & 4.5 & & \\
\hline 2 & PRAKM & $\mathbf{V}$ & -19.75 & & .5 & & \\
\hline 3 & PPR . & $\mathbf{H}$ & -16.50 & & & & \\
\hline 4 & PPR & $\mathbf{V}$ & 22 & & & & \\
\hline 5 & PRAKM & $\mathrm{H}$ & -9 & & & & \\
\hline 6 & PRAKM & V & -12.5 & & 0 & -21.5 & \\
\hline 7 & PPR & $\mathbf{H}$ & 15.5 & 3 & & & \\
\hline 8 & PPR & V & -8.5 & & 6 & 11 & 9.5 \\
\hline 9 & PRAKM & $\mathrm{H}$ & -2.75 & & -37 & & -33 \\
\hline 10 & PRAKM & V & -34.25 & & 12.5 & & 12 \\
\hline 11 & PPR & $\mathbf{H}$ & -19.5 & & & -8.5 & \\
\hline 12 & PPR & V & 20 & & & -3 & \\
\hline
\end{tabular}

staircase means. The means obtained (predominantly convergent and downturned) are also consistent with previous findings (Levy, 1969).

Forty-eight separate SPRT decisions were included in the main design, plus six reruns following SPRT rejections of $\mathrm{H}_{\mathrm{O}}$ on stability tests. The null hypothesis was always accepted in these six SPRTs. Nine identity SPRTs also rejected $\mathrm{H}_{0}$. Table 2 shows the estimated error in the 15 cases where $\mathrm{H}_{\mathrm{O}}$ was rejected.

Fifteen $\mathrm{H}_{\mathrm{O}}$ rejections in 54 tests is more than can be due to chance alone, so they must be considered in detail. Many $\mathrm{H}_{0}$ rejections may be due to experimental error, especially as all but five cases are less than $11^{\circ}$ (twice the measurement error). Four sources of error may be considered.

The two tasks differed in one important way. In PPR, the tixation point was present for only $2 \mathrm{sec}$ in 25. In PRAKM. it was there for 20. Levy (1968) presents many instances where small variation in fixation conditions affect these measures. When eye position is not controlled, as in the PPR mode, systematic biases and aftereffects can occur. Even though subjects tried to keep looking in the same direction, this effort would be of small help (Levy, 1968; Skavenski \& Steinman, 1970).

In the tirst session, the original setting up. measurement, long runs, and remeasurement when the first stability test failed meant that several sessions lasted over $3 \mathrm{~h}$. The first identity test, which came at the end. may well be biased by fatigue. Six $\mathrm{H}_{\mathrm{O}}$ rejections occurred then, which is more than in any other SPRT. Unfamiliarity may also be a factor in identity tests. especially the first. since these were the only occasions where subjects experienced the alternative task. The effect was never strong because the stimulus was placed at the predicted position of maximum uncertainty.

Finally. some subjects showed strong sequential dependencies when the relation between responses on trials $\mathrm{N}$ and $\mathrm{N}-1 \mathrm{w}$ as tested by Tocher's modification of the Fisher exact probability ratio test $(a=0.05, \beta$ varies with sample size) (Siegal, 1956), Eleven signiticant cases occurred, when three were expected by chance. All were chaining biased, as were the majority of all cases. As can be seen in Table 3, these biases are subject specific, and associated with accepting $\mathrm{H}_{\mathrm{O}}$, though in extremes they would cause the opposite. These four subjects altogether account for three SPRT rejections of $\mathrm{H}_{0}$, which means that they differ little from other subjects and excess Type 2 errors are unlikely. When interpreting the results, the presence of these and other response biases and departures from assumptions must be kept in mind.

The SPRT design has confirmed and extended the results of the pilot studies. Errors of localization of successively tlashed points and AKI direction reports are reliable and highly correlated. We can also say that they are stable. sometimes for periods well over a week. in 24 cases out of 30 and identical in 15 cases out of 24 . In 9 of the remaining 15 cases, the degree of

Table 3

Cases Where Significant Chaining Bias Was Found, Indicated by the Decision Which Eventually Was Made

\begin{tabular}{|c|c|c|c|c|c|c|}
\hline \multicolumn{3}{|c|}{ Preliminary Staircase } & \multicolumn{4}{|c|}{ SPRT } \\
\hline Subject & Task & $\begin{array}{c}\text { Dimen- } \\
\text { sion }\end{array}$ & $\begin{array}{c}\text { First } \\
\text { Sta- } \\
\text { bility }\end{array}$ & $\begin{array}{l}\text { First } \\
\text { Iden- } \\
\text { tity }\end{array}$ & $\begin{array}{l}\text { Second } \\
\text { Sta- } \\
\text { bility }\end{array}$ & $\begin{array}{l}\text { Second } \\
\text { Iden- } \\
\text { tity }\end{array}$ \\
\hline 1 & PRAKM & $\mathbf{H}$ & & & & \\
\hline 2 & PRAKM & V & $\mathbf{H}_{\mathbf{o}}$ & & & \\
\hline 3 & PPR & H & $\mathrm{H}_{\mathrm{o}}$ & $\mathrm{H}_{\mathrm{o}}$ & $\mathbf{H}_{\mathrm{O}}$ & $\mathrm{H}_{\mathrm{o}}$ \\
\hline 4 & PPR & V & & & & \\
\hline 5 & PRAKM & $\mathbf{H}$ & & & & \\
\hline 6 & PRAKM & $\mathrm{V}$ & & & & \\
\hline 7 & PPR & $\mathbf{H}$ & & $\mathbf{H}_{\mathrm{o}}$ & $\mathbf{H}_{\mathrm{o}}$ & $\mathrm{H}_{\mathrm{o}}$ \\
\hline 8 & PPR & V & & & & \\
\hline 9 & PRAKM & $\mathbf{H}$ & & & & \\
\hline 10 & PRAKM & V & & & & \\
\hline 11 & PPR & H & $\mathrm{H}_{\mathbf{o}}$ & $\mathrm{H}_{\mathrm{o}}$ & $\mathrm{H}_{\mathrm{o}}$ & \\
\hline 12 & PPR & V & & & & \\
\hline
\end{tabular}

Note-Chaining is subject specific and common. Alternation seldom occurred and never significantly. 
difference or shift was less than twice the error of measurement (119). Whether they would be more stable with less measurement error or more often identical in conditions where eye position is more rigidly controlled remains an open question.

However, five of the $\mathrm{H}_{0}$ rejections cannot be disregarded even after all sources of error are considered. The identity hypothesis is consistently rejected in two subjects (Nos. 9 and 10). and the stability hypothesis clearly does not hold in one case (No. 6). We must concluded that in the conditions of this experiment PRAKM and PPR are usually, but not always, stable and identical. Therefore, in some individuals, the slow components of drift. presumed to cause both effects, cannot be a permanent feature of the oculomotor system. but must vary with both stimulus conditions and time.

\section{DISCUSSION}

Most cases of apparent movement are adequately explained by the assumptions that the directionally biased drifts which occur constantly are not taken into account to the same degree as saccades. Usually an assumption of differential sensitivity is sufficient, but there are a small class of experiments where apparent movement occurs without any correlated eye movement at all. which not only support the outflow theory. but require it to be extended by separating drift into monitored and unmonitored components which cancel each other (Levy, 1972).

Ludvigh (1952) had recognized the need for "parametric adjustment." an unconscious reflex loop in the oculomotor system. now known to be a general pattern of muscle spindle action (Hammond, Merton, \& Sutton. 1956). When Skavenski and Steinman (1970) discovered that they could maintain fixation on a point without saccades, they recognized that there must be a slow response, opposing the normal drift movements, which they called "corrective drift." When Levy (1972) learned from Steinman (personal communication. 1969) that the AKI movement still occurred as normal in this sort of viewing, and that in many situations apparent movement still occurred after nystagmus was suppressed (Graybiel \& Hupp. 1946). he argued that there must be some "compensatory pursuit-like outflow which just cancelled the drift" and which must be at least partially monitored, since the fixation point appeared to be moving even though the subject's eye was not. Stoper (1973) has some evidence that pursuits are at least partially monitored. The only difference between this compensatory pursuit-like outflow and other pursuits is that it is a response which tracks the apparent movement of the visual field caused by transient disturbances within the oculomotor system, so that there is no movement of the eyeball itself relative to the head. At first it merely maintains fixation more precisely, is voluntary, and at least partially monitored. The initial apparent movement fades to an extent which depends on past experience, the visual surround, and the nature of the disturbance (Graybiel \& Hupp, 1946). Possibly at later stages, other unmonitored slow responses take over.

Many systems cause slow responses and some presumably amalgamate to determine the direction of drift towards the PPR. Fundamental mechanical forces arise from the elastic tissues in the muscles and other structures in the orbit (Adler, 1959; Duke-Elder, 1949; Skavenski \& Steinman, 1970). There are inputs from the neck and vestibular systems (Adler, 1959). Lock-up mechanisms potentiate muscles, producing increased tension in the direction of previous turn, particularly when that turn is a version (Ebenholtz \&Wolfson, 1975; Levy, 1973). Fatigue processes can also decrease tension, particularly following prolonged stress in susceptible subjects (Levy, 1973). Other slow responses include compensatory pursuit-like outflow, vergence, accommodative vergence, and simple pursuits. They depend to some extent on visual input and may not affect the PPR.

All these causes of slow movements are components of drift. They can differ both in direction and the degree to which they are monitored. Any one eye position can be the result of several different mixtures and cause different direction of apparent movement (Graybiel \& Hupp, 1946; Levy, 1968, 1973). Although they are sufficiently stable features of the oculomotor system to explain the reliability of AKI direction reports and the correlation of PRAKM and PPR, they can vary. The cause of this variability affects ocular posture and so deserves more study.

\section{REFERENCES}

Adnms. H. F. Autokinetic sensations. Psychological Monograph, 1912, 14, 1-45.

Adler, F. H. Physiology of the eye. St. Louis: Mosby, 1959.

Armitage, P. Sequential medical trials. Oxford: Blackwells, 1960.

CARR, H. A. A visual illusion of motion during eye closure. Psychological Review Monograph Supplement, 1907, 7, whole issue.

CARr. H. A. The autokinetic sensation. Psychological Review, 1910, 17. 42-75.

Duke-Elder, W. S. Text book of Ophthalmology (Vol. IV) The neurology of vision. Motor and optical anomalies. London: Kimpton, 1949.

Ebenholtz, S. M. \& Wolfson, D. M. Perceptual aftereffects of sustained convergence. Perception \& Psychophysics, 1975, 17 , 485-491.

Ginsborg, B. L., \& Maurice. D. M. Involuntary movements of the eye during fixation and blinking. British Joumal of Ophthalmology, 1959, 43, 435-437.

GraYbiel, A., \& HuPp, D. The oculogyral illusion: A form of apparent motion which may be observed following stimulation of semicircular canals. Journal of Aviation Medicine, 1946. 17. 3.27. 
Hammono. P. H. Merton, P, A.. \& Sutton, G. G. Nervous gradation of muscular contraction. British Medical Bulletin. 1956. 12, 214-218.

LEVy, J. Measurement and explanation of the directional component of the autokinetic illusion. MSc thesis, Monash University, Melbourne, Australia, 1966.

LeVY, J. Physiological position of rest and phoria. American Journal of Ophthalmology, 1969, 68, 706-713.

LEVY. I. The autokinetic illusion: A systematic review of measures. theories, and dependent variables. Psychological Bullerin, 1972, 73, 457-474.

LEVY, J. Autokinesis direction during and after eye turn. Perception \& Psychophysics, 1973, 13, 337-343.

Ludvigh. E. Control of ocular movements and visual interpretation of environment. AMA Archives of Opthalmology. 1952. 48. $442-448$.

Matin. L.. \& Kiblek. G. Acuity of visual perception of direction in the dark for various positions of the eye in the orbit. Perceptual and Motor Skills, 1966, 22. 407-420.

Matin. L.. Pearce, D., Matin, E., \& Kibler, G. Visual perception of direction in the dark. Roles of local sign. eye movement, and ocular proprioception. Vision Research. 1966. 6. $453-469$.

NaChImas, J. Determiners of drift of the eye during monocular fixation. Journal of the Optical Society of America, 1961 . 49. 901.908 .

Pearce. D. Consistency of individual patterns of autokinetic direction. Perceptual and Motor Skills, 1966, 23, 1119-1123.

SIEGAL.S. Non-parametric statistics for the behavioral Sciences. New York: McGraw-Hill, 1956.

Skavenski. A.. \& Steinman, R. M. Control of eye position in the dark. Vision Research, 1970, 10. 193-203.
Stoper. A. E. Apparent motion of stimuli presented stroboscopicaliy during pursuit movement of the eye. Perception \& Psychophysics, 1973, 13. 201-211.

Wetherili, G. B. Sequential methods in statistics. London: Methuen, 1906

\section{NOTES}

1. Matin et al. (1966) compared the judgment error with the target's retinal position at the time of judgment. thereby disregarding the lew saccades that occurred in theis oftuation. If they had distinguished drifts. which are probably unmonitored and contribute to the error, from saccades, which are monitored and do not, then they may have obtained an even greater relationship between eye movement. local sign, and localization errors.

2. It is now clear, both from later experience (Levy. 1973) and theoretical and statistical considerations, that 2-deg steps would substantially reduce PRAKM aftereffect biases and measurement error. Data from Matin and Kibler (1966) suggest that PPR error mav, in some situations. be less than $1 / 2 \mathrm{deg}$. Step size could not be halved as Wetherill (1966) recommended because of the perimeter's limitation to 5 -deg minimum steps.

3. The author is indebted to Dr. Max Coltheart for suggesting this approach to the problem.

(Received for publication April 1974; revision received July 1975 .) 